Swarthmore College

Works

$5-17-2005$

\title{
Correlation Length And Chirality Of The Fluctuations In The Isotropic Phase Of Nematic And Cholesteric Liquid Crystals
}

Jacob J. Krich , '00

Mark B. Romanowsky , '03

Peter J. Collings

Swarthmore College, pcollin1@swarthmore.edu

Follow this and additional works at: https://works.swarthmore.edu/fac-physics

Part of the Physics Commons

Let us know how access to these works benefits you

\section{Recommended Citation}

Jacob J. Krich , '00; Mark B. Romanowsky , '03; and Peter J. Collings. (2005). "Correlation Length And Chirality Of The Fluctuations In The Isotropic Phase Of Nematic And Cholesteric Liquid Crystals". Physical Review E. Volume 71, Issue 5. DOI: 10.1103/PhysRevE.71.051712

https://works.swarthmore.edu/fac-physics/131

This work is brought to you for free by Swarthmore College Libraries' Works. It has been accepted for inclusion in Physics \& Astronomy Faculty Works by an authorized administrator of Works. For more information, please contact myworks@swarthmore.edu. 


\title{
Correlation length and chirality of the fluctuations in the isotropic phase of nematic and cholesteric liquid crystals
}

\author{
Jacob J. Krich, ${ }^{*}$ Mark B. Romanowsky, ${ }^{*}$ and Peter J. Collings \\ Department of Physics and Astronomy, Swarthmore College, Swarthmore, Pennsylvania 19081, USA
}

(Received 24 January 2005; published 17 May 2005)

\begin{abstract}
Light-scattering measurements of the correlation length in the isotropic phase of a nematic liquid crystal reveal a temperature dependence following Landau-de Gennes theory for the isotropic phase with a bare correlation length smaller than has been measured in other liquid crystals. Similar measurements in a cholesteric liquid crystal demonstrate that the correlation length in the isotropic phase is larger than typically found in nematics and that the chirality of the fluctuations in the isotropic phase is slightly higher than the chirality of the cholesteric phase. Landau-de Gennes theory of the cholesteric phase describes the chirality in the cholesteric phase well but predicts that the chirality in the isotropic phase is temperature independent, which is not consistent with the data. There is a discontinuity in the chirality at the cholesteric-isotropic transition of about $15 \%$, which is less than the predictions of Landau-de Gennes theory but more than the typical specific volume discontinuity at transitions to the isotropic phase. Except for a mismatch in the discontinuities at the transition, the chirality data resemble the temperature behavior of variables just below a critical point, in spite of the fact that this system is far from a critical point.
\end{abstract}

DOI: 10.1103/PhysRevE.71.051712

PACS number(s): 61.30.Cz, 61.30.Gd, 64.70.Md

\section{INTRODUCTION}

Advances in the understanding of phase transitions have highlighted the central role fluctuations play in all but the most strongly discontinuous transitions. Phase transitions in thermotropic liquid crystals are an excellent example of this because the transitions are weakly discontinuous or continuous. In addition, some liquid crystals are chiral, and recent theoretical and experimental studies have pointed out the strong effect chirality can have on the nature of a phase transition. Yet there is limited quantitative information on the fluctuations near some thermotropic liquid-crystal phase transitions. For example, one of the most important parameters in describing a phase transition is the bare correlation length, since this is the length scale that is renormalized as the phase transition is approached. Measurements of the correlation length in the isotropic phase are few, having been done some time ago and almost exclusively on one nonchiral system [1-3]. Yet in many cases theorists must use a value for the bare correlation length in order to make comparisons between theory and experiment. Clearly, additional measurements of the bare correlation length, especially in thermotropic systems in which the molecules are quite different from the ones studied in the past, are necessary. An interesting recent development is that the measurement of the bare correlation length in a lyotropic chromonic nematic liquid crystal, in which the ordering entities are aggregates of molecules instead of molecules, has been reported [4]. The bare correlation length in this system is three to four times longer than the measurements in thermotropic nematic systems.

A more important example concerns measurements of chirality near phase transitions. The long-range orientational

\footnotetext{
*Present address: Department of Physics, Harvard University, Cambridge, MA 02138, USA.
}

order of cholesteric liquid crystals makes the measurement of chirality in the cholesteric phase easy. Measurement of the chirality of the fluctuations in the isotropic phase near the transition is very difficult. In fact, only one such measurement has been done, and that was performed by Koistenen and Keyes on a system with a continuous transition from the isotropic phase to a more ordered isotropic liquid-crystal phase (Blue Phase III) [5]. No measurement of the chirality of the fluctuations in the isotropic phase near the phase transition to the cholesteric phase has been done, and no theory specifically describing the chirality across the cholestericisotropic liquid transition has been formulated. Yet chirality has been the subject of many theoretical efforts [6-15], and some understanding of the behavior of chirality on both sides of this transition should be possible. But at this time a good understanding is lacking. In fact, when comparing theories of the isotropic phase to experimental data, the chirality of the cholesteric phase must be used because the chirality of the isotropic phase is unknown.

In an effort to check the experimental procedures utilized in this investigation and to probe the bare correlation length in a nonchiral system quite different from those studied previously, light-scattering measurements in the isotropic phase of p-pentyl-p'-cyanobiphenyl (5CB) were performed. These measurements are difficult because the correlation length is much less than the wavelength of light in the material. These results demonstrate that the bare correlation length in this smaller, polar molecular system is less than in systems composed of larger, nonpolar molecules. An interesting aspect of these experiments is that the very large electric susceptibility anisotropy of 5CB produces strong enough light scattering for the detected intensity to have a contribution from multiple scattering. This is taken into account by assuming that single and double scattering are the only contributions that need to be included in the analysis.

The most significant results concern the measurements of chirality across the cholesteric-isotropic transition in a mix- 
ture of $35 \%$ chiral and $65 \%$ racemic S-4-(2methylbutyl)phenyl-4-decyloxybenzoate (CE6 and CE6R). The chirality increases with temperature in both phases, with a relatively small discontinuity at the phase transition. This behavior is similar to what one observes for the specific volume across a liquid crystal to isotropic liquid transition, but the discontinuity in the chirality is much greater. The measurements in the isotropic phase follow the predictions of Landau-de Gennes theory for short range order in the isotropic phase of a chiral system, but in this theory chirality is an independent parameter with no indication of how it depends on temperature. The temperature dependence of the chirality in the cholesteric phase can be understood by modified mean-field theories of the Landau-de Gennes type, but these predict that the chirality in the isotropic phase is independent of temperature while the data indicate otherwise. This discrepancy is not surprising, since chirality is not related in a direct way to the orientational order parameter used in these theories. A theory developed for the critical point that occurs in some chiral liquid-crystal systems agrees with the chirality measurements in both the cholesteric and isotropic phases except for the size of the discontinuity, even though these measurements are from a region of the phase diagram far from the critical point. But most importantly, these results present how much change in chirality occurs at the cholesteric-isotropic transition in a typical system.

\section{THEORY}

The theory behind light scattering in the isotropic phase of liquid crystals was worked out some time ago and has been used in many investigations. A formulation that is general enough for both nonchiral and chiral liquid crystals utilizes a scattering Mueller matrix in which the elements $\left\langle\mu_{i j}\right\rangle$ depend on the mean-square amplitudes of the fluctuating basis modes and the scattering angle [16],

$$
\begin{gathered}
\left\langle\mu_{11}\right\rangle=2\left\langle\epsilon_{0}^{2}\right\rangle\left(c^{2}+1\right)^{2} / 3+2\left\langle\epsilon_{0}^{2}\right\rangle / 3+\left(\left\langle\epsilon_{2}^{2}\right\rangle+\left\langle\epsilon_{-2}^{2}\right\rangle\right)\left(s^{2}+1\right)^{2} \\
+2\left(\left\langle\epsilon_{1}^{2}\right\rangle+\left\langle\epsilon_{-1}^{2}\right\rangle\right) c^{2} \\
\left\langle\mu_{12}\right\rangle=\left\langle\mu_{21}\right\rangle=2\left\langle\epsilon_{0}^{2}\right\rangle\left(c^{2}+1\right)^{2} / 3-2\left\langle\epsilon_{0}^{2}\right\rangle / 3 \\
-\left(\left\langle\epsilon_{2}^{2}\right\rangle+\left\langle\epsilon_{-2}^{2}\right\rangle\right)\left(1-s^{4}\right) \\
\left\langle\mu_{13}\right\rangle=\left\langle\mu_{31}\right\rangle=\left\langle\mu_{23}\right\rangle=\left\langle\mu_{32}\right\rangle=\left\langle\mu_{34}\right\rangle=\left\langle\mu_{43}\right\rangle=0 \\
\left\langle\mu_{14}\right\rangle=\left\langle\mu_{41}\right\rangle=-2\left(\left\langle\epsilon_{2}^{2}\right\rangle-\left\langle\epsilon_{-2}^{2}\right\rangle\right) s\left(s^{2}+1\right) \\
\left\langle\mu_{22}\right\rangle=2\left\langle\epsilon_{0}^{2}\right\rangle\left(c^{2}+1\right)^{2} / 3+2\left\langle\epsilon_{0}^{2}\right\rangle / 3+\left(\left\langle\epsilon_{2}^{2}\right\rangle+\left\langle\epsilon_{-2}^{2}\right\rangle\right) c^{4}-2\left(\left\langle\epsilon_{1}^{2}\right\rangle\right. \\
\left.+\left\langle\epsilon_{-1}^{2}\right\rangle\right) c^{2} \\
\left\langle\mu_{24}\right\rangle=\left\langle\mu_{42}\right\rangle=2\left(\left\langle\epsilon_{2}^{2}\right\rangle-\left\langle\epsilon_{-2}^{2}\right\rangle\right) s c^{2} \\
\left\langle\mu_{33}\right\rangle=-4\left\langle\epsilon_{0}^{2}\right\rangle\left(c^{2}+1\right) / 3+2\left(\left\langle\epsilon_{1}^{2}\right\rangle+\left\langle\epsilon_{-1}^{2}\right\rangle\right) c^{2} \\
\left\langle\mu_{44}\right\rangle=-4\left\langle\epsilon_{0}^{2}\right\rangle\left(c^{2}+1\right) / 3+4\left(\left\langle\epsilon_{2}^{2}\right\rangle+\left\langle\epsilon_{-2}^{2}\right\rangle\right) s^{2}-2\left(\left\langle\epsilon_{1}^{2}\right\rangle\right. \\
\left.+\left\langle\epsilon_{-1}^{2}\right\rangle\right) c^{2}
\end{gathered}
$$

where $s$ and $c$ are the sine and cosine of half the scattering angle $\theta$, respectively. Factors involving the average electric susceptibility and the phases of the basis modes are not included in Eq. (1) because they do not contribute to the scattering intensity. The $\left\langle\epsilon_{m}\right\rangle$ are the spherical basis modes of the electric susceptibility anisotropy tensor in which one mode with mean-square amplitude $\left\langle\epsilon_{0}^{2}\right\rangle$ describes a uniaxial director field, two modes with mean-square amplitudes $\left\langle\epsilon_{1}^{2}\right\rangle$ and $\left\langle\epsilon_{-1}^{2}\right\rangle$ describe conical spiral director fields (opposite senses), and two modes with mean-square amplitudes $\left\langle\epsilon_{2}^{2}\right\rangle$ and $\left\langle\epsilon_{-2}^{2}\right\rangle$ describe planar spiral director fields (opposite senses). The intensity of scattered light is found by applying the scattering Mueller matrix to the incident polarization Stokes vector and then applying the proper Mueller matrix to this result to take into account the optical elements between the sample and the detector. For example, if vertically polarized light is incident on the sample and only vertically polarized light is allowed to reach the detector (the scattering plane is horizontal), then the intensity at the detector is given by

$$
\begin{aligned}
I_{\mathrm{VV}}= & \frac{I_{0}}{2}\left(\left\langle\mu_{11}\right\rangle+\left\langle\mu_{22}\right\rangle-2\left\langle\mu_{12}\right\rangle\right) \\
= & \frac{I_{0}}{2}\left[\frac{8}{3}\left\langle\epsilon_{0}^{2}\right\rangle+4\left(\left\langle\epsilon_{2}^{2}\right\rangle+\left\langle\epsilon_{-2}^{2}\right\rangle\right)\right],
\end{aligned}
$$

where $I_{0}$ is proportional to the incident intensity. If righthanded circularly polarized light is incident on the sample and only right-handed circularly polarized light is detected, then the intensity is given by

$$
\begin{aligned}
I_{\mathrm{RR}}= & \frac{I_{0}}{2}\left(\left\langle\mu_{11}\right\rangle+2\left\langle\mu_{14}\right\rangle+\left\langle\mu_{44}\right\rangle\right) \\
& =\frac{I_{0}}{2}\left[\frac{2}{3} c^{4}\left\langle\epsilon_{0}^{2}\right\rangle+(s-1)^{4}\left\langle\epsilon_{2}^{2}\right\rangle+(s+1)^{4}\left\langle\epsilon_{-2}^{2}\right\rangle\right] .
\end{aligned}
$$

The mean-square amplitude of the spherical susceptibility anisotropy modes can be determined using the Landau-de Gennes real-space expression for short-range order in the isotropic phase, converting it to momentum space, and then using the equipartition theorem to describe the energy associated with each mode [16]. The free-energy density in momentum space can be written

$$
\Phi_{\mathrm{V}}=\frac{1}{2} A \sum_{m}\left[a-m b q_{0} q+\left(b+\frac{d}{6}\left(4-m^{2}\right)\right) q^{2}\right]\left\langle\epsilon_{m}^{2}(q)\right\rangle
$$

where $q$ is the wave vector; $m$ is the index for the five basis modes; $a, b$, and $d$ are the coefficients of terms in the realspace free-energy density; and $q_{0}$ is another coefficient of the free-energy density term representing the chirality of the system $\left(q_{0}=4 \pi / P\right.$, where $P$ is the pitch of the director helix). $q_{0}$ is negative for a right-handed helix and positive for a lefthanded helix. $A$ is a proportionality constant. The equipartition theorem yields an expression for the mean-square amplitude of each of the modes 


$$
\left\langle\epsilon_{m}^{2}(q)\right\rangle=\frac{B k_{\mathrm{B}} T}{a-m b q_{0} q+\left[b+\frac{d}{6}\left(4-m^{2}\right)\right] q^{2}},
$$

where $k_{B}$ is the Boltzmann constant, $T$ is the absolute temperature, and $B$ is a proportionality constant.

Substituting the expressions for the $\left\langle\mu_{i j}\right\rangle$ into the intensity equation and then using the mean-square amplitudes of the basis modes gives the following relations for the two cases discussed earlier:

$$
\begin{aligned}
I_{\mathrm{VV}}= & \frac{C}{T-T^{*}}\left[\frac{\frac{2}{3} k_{\mathrm{B}} T}{1+\left(\xi_{1}^{2}+\frac{2}{3} \xi_{2}^{2}\right) q^{2}}+\frac{k_{\mathrm{B}} T}{1-\xi_{1}^{2} q_{0}^{2}+\xi_{1}^{2}\left(q-q_{0}\right)^{2}}\right. \\
& \left.+\frac{k_{\mathrm{B}} T}{1-\xi_{1}^{2} q_{0}^{2}+\xi_{1}^{2}\left(q+q_{0}\right)^{2}}\right], \\
I_{\mathrm{RR}}= & \frac{D}{T-T^{*}}\left[\frac{\frac{2}{3} c^{4} k_{\mathrm{B}} T}{1+\left(\xi_{1}^{2}+\frac{2}{3} \xi_{2}^{2}\right) q^{2}}+\frac{\left(s^{2}-1\right)^{4} k_{\mathrm{B}} T}{1-\xi_{1}^{2} q_{0}^{2}+\xi_{1}^{2}\left(q-q_{0}\right)^{2}}\right. \\
& \left.+\frac{\left(s^{2}+1\right)^{4} k_{\mathrm{B}} T}{1-\xi_{1}^{2} q_{0}^{2}+\xi_{1}^{2}\left(q+q_{0}\right)^{2}}\right]
\end{aligned}
$$

where $\xi_{1}^{2}=b / a$ and $\xi_{2}^{2}=d / a$ are squared correlation lengths. $C$ and $D$ are proportionality constants, and $a=a_{0}\left(T-T^{*}\right)$, where $a_{0}$ is a constant and $T^{*}$ is the temperature at which the nonchiral fluctuations in the isotropic phase diverge (if not interrupted by the first-order phase transition). $\xi_{1}$ describes the size of the correlated regions, and $\xi_{2}$ is related to how elliptical they are. There is some evidence that $\xi_{2}<\xi_{1}$ [3], making it convenient to set $\xi_{2}=0$. The scaling of the correlation length as the transition is approached is then given by

$$
\xi_{1}=\sqrt{\frac{b T^{*}}{a_{0} T^{*}\left(T-T^{*}\right)}}=\xi_{0} \sqrt{\frac{T^{*}}{T-T^{*}},}
$$

where $\xi_{0}=\sqrt{b /\left(a_{0} T^{*}\right)}$ is the bare correlation length.

The Landau-de Gennes phenomenological theory of the nematic liquid-crystal phase can be extended to include chirality by simply adding terms allowed by symmetry to the nonchiral free-energy expression. There are several terms that are good candidates, but knowing which ones are likely to dominate is difficult to ascertain. Since the results are qualitatively the same regardless of which terms are added, one example is given here that has been successful in describing the chirality of a cholesteric phase [17]. The free energy of a chiral system can be written

$$
f=\frac{1}{3}\left[a_{0}\left(T-T^{*}\right)-\frac{3}{4} b q_{0}^{2}\right] S^{2}-\frac{2}{27} \mu S^{3}+\frac{1}{9} \lambda S^{4},
$$

where $a_{0}, b, \mu$, and $\lambda$ are temperature-independent constants, $S$ is the orientational order parameter, and $q_{0}$ is the chirality. Since it is not known how the chirality depends on the order parameter, both linear and nonlinear dependences are considered,

$$
q_{0}=q_{\mathrm{I}}\left(1+\alpha S^{\beta}\right),
$$

where $\alpha$ and $q_{I}$ are constants and $\beta$ equals 1,2 , or 3. For a given value of $\beta$, the free energy can be minimized as a function of temperature for any set of parameters $T^{*}, a_{0}, b$, $\mu, \lambda, q_{\mathrm{I}}$, and $\alpha$. Note that this form of $q_{0}$ introduces terms in the free energy of the form $q_{\mathrm{I}}^{2} S^{2}, q_{\mathrm{I}}^{2} S^{\beta+2}$, and $q_{\mathrm{I}}^{2} S^{2 \beta+2}$. Note also that $q_{\mathrm{I}}$ is the temperature-independent chirality of the isotropic phase. Since the parameters in the free energy are not known, they must be determined by a fit to experimental data.

Since this theory is based on an orientational order parameter, it can only describe the chirality through its dependence on the order parameter. This clearly creates problems. For example, if this theory is fit to experimental data on the chirality in the cholesteric phase in a helix inversion system, the prediction of the chirality in the isotropic phase where the order parameter is zero does not even match the sense of chirality in the isotropic phase (which was all that could be determined experimentally) [17]. So rather than treating the cholesteric and isotropic phases as very different due to a large change in the order parameter, perhaps a theory that begins with the idea that the liquid-crystal and isotropic phases are similar (because of the existence of a nearby critical point) can better describe how the chirality changes in crossing this transition even though the system is not near a critical point. Such a theory was first proposed by Lubensky and Stark [13] and further generalized and tested by others [14].

This theory defines the order parameter as

$$
\langle\phi\rangle=\langle\psi\rangle-\langle\psi\rangle_{\mathrm{c}}=\langle(\boldsymbol{\nabla} \times \mathbf{Q}) \cdot \mathbf{Q}\rangle-\langle\psi\rangle_{\mathrm{c}},
$$

where $\langle\psi\rangle_{\mathrm{c}}$ is the value of $\langle(\boldsymbol{\nabla} \times \mathbf{Q}) \cdot \mathbf{Q}\rangle$ at the critical point and $\mathbf{Q}$ is the thermally fluctuating alignment tensor. Assuming this order parameter obeys the normal scaling relation in the vicinity of the critical point, $\langle\phi\rangle$ must obey a simple cubic equation involving the two scaling fields (ordering and nonordering). These two scaling fields are assumed to be linear combinations of the temperature and chiral fraction. Comparison of this theory with precise heat capacity measurements in the vicinity of a critical point allowed the temperature and chiral fraction dependence of the two scaling fields to be determined for one chiral-racemic cholesteric system, thus revealing how $\langle\phi\rangle$ varied with temperature at a fixed chiral fraction [18]. The order parameter is continuous in the supercritical region, but is discontinuous in passing through the transition line that ends at the critical point. The variation of the order parameter with temperature at a fixed chiral fraction is not symmetric on either side of the transition. This theory can be applied to other cholesteric systems; all that needs to be specified is how far below the critical chiral fraction the system under study is. 


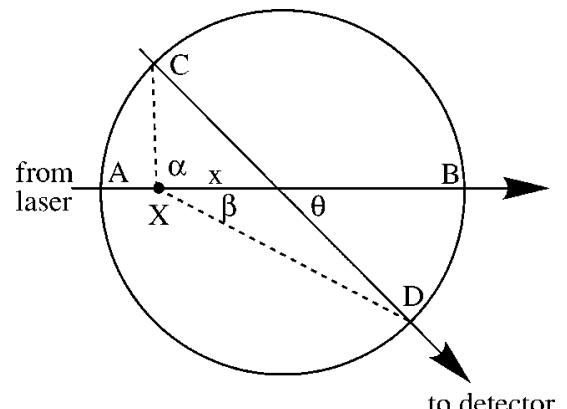

FIG. 1. Geometry for calculating the contribution from double scattering. The scattering angle is $\theta$, the first scattering event occurs at point $X$, and the second scattering event occurs somewhere along line $C D$. The probability of double scattering is proportional to the angle $\alpha+\beta$. This angle is then averaged for all points along line $A B$ a distance $x$ from the center.

\section{EXPERIMENT PROCEDURES AND RESULTS}

The first material studied was the nematic liquid crystal $5 \mathrm{CB}$, purchased from BDH Limited and used without additional purification except filtering through a $0.2 \mu \mathrm{m}$ nylon filter. The nematic-isotropic phase transition occurs around $308 \mathrm{~K}$, therefore measurements were conducted over a $15 \mathrm{~K}$ range of temperature higher than this value. Measurements of the intensity of light scattering as a function of scattering wave vector, $q=\left(4 \pi n / \lambda_{0}\right) \sin (\theta / 2)$, where $n$ is the index of refraction of the sample, were recorded using a cylindrical sample vessel (diameter of $1 \mathrm{~cm}$ ) and vertical polarizers in the input beam and in front of the detector. The sample vessel was surrounded by index matching fluid and the incident $25 \mathrm{~mW}$ light with $\lambda_{0}=488 \mathrm{~nm}$ came from an argon-krypton laser. The temperature was controlled by circulating temperature-regulated water through a coil in the indexmatching fluid. The alignment of the system was adjusted until scattering from the index-matching fluid was constant over the entire range of scattering angles with no sample vessel present.

It was quickly noted that the data contained an additional contribution when compared to the light-scattering results from many other liquid crystals. Because 5CB has a much larger electric susceptibility anisotropy than typical liquid crystals, it is likely that the amount of scattering from the fluctuations is high enough to cause a larger contribution from multiple scattering effects. To check whether this is the case or not, a simple calculation of the angular dependence due to double scattering was performed. This calculation is made simpler by the approximation that each scattering event is isotropic, i.e., the angular dependence of the double scattering contribution comes predominantly from geometric effects and not from the angular dependence of Eq. (6). This approximation is valid since the correlation length is much less than the wavelength of light used for the scattering. As can be seen from Fig. 1, for double scattering to be detected, the first scattering event must occur along the input laser beam (line $A B$ in Fig. 1) and the second scattering event must occur along the acceptance beam of the detector optics (line $C D$ in Fig. 1). If the point of the first scattering event is located a distance $x$ from the center of the cylindrical sample

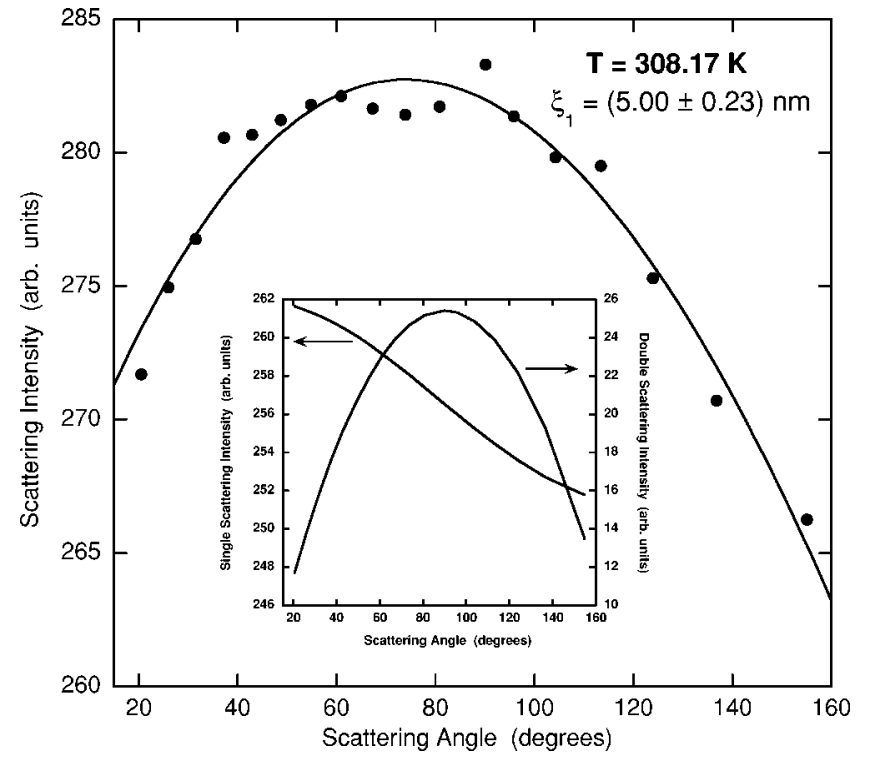

FIG. 2. Scattering intensity as a function of scattering angle for $5 \mathrm{CB}$. The inset shows the contributions from single and double scattering resulting from the fitting procedure described in the text that results in a correlation length of $5.00 \pm 0.23 \mathrm{~nm}$.

vessel (point $X$ in Fig. 1), then the probability of a second scattering event along line $C D$ is proportional to the angle $\alpha+\beta$ subtended by line $C D$ at point $X$. Averaging $\alpha+\beta$ for all $X$ on line $A B$ gives a weakly angular-dependent function with a shallow minimum at $\theta=90^{\circ}$. Changes in the polarization during the two scattering events do not affect the calculation, and there should not be a strong change in the scattering volume with angle. Thus double scattering shows up as a contribution that is almost independent of angle, but because the measured intensity is multiplied by $\sin \theta$ to compensate for the change in scattering volume for single scattering, the double scattering contribution strongly depends on angle and peaks at $90^{\circ}$.

The single scattering contribution is given by Eq. (6) with $q_{0}=0$. For simplicity, $\xi_{2}$ is assumed to be zero, so

$$
I_{\mathrm{VV}}=\frac{C T}{\left(T-T^{*}\right)\left(1+\xi_{1}^{2} q^{2}\right)},
$$

where $C$ is a new proportionality constant. When a function containing the expressions for both single and double scattering, each with an adjustable coefficient, is fit to the lightscattering data ( $\xi_{1}$ is the only other fitting parameter), results as shown in Fig. 2 are obtained. The graph contains the actual data and fitting result, whereas the inset shows the magnitude of the two contributions as a function of scattering angle. Note that the contribution from single scattering dominates, but that the small value of the correlation length means that the majority of the change with scattering angle comes from the double scattering contribution.

This procedure was performed for 11 different temperatures above the nematic to isotropic phase transition. The data are graphed in Fig. 3 where the theoretical dependence of Eq. (8) has been fit to the data. The resulting value for the bare correlation length $\xi_{0}$ is $0.40 \pm 0.03 \mathrm{~nm}$. 


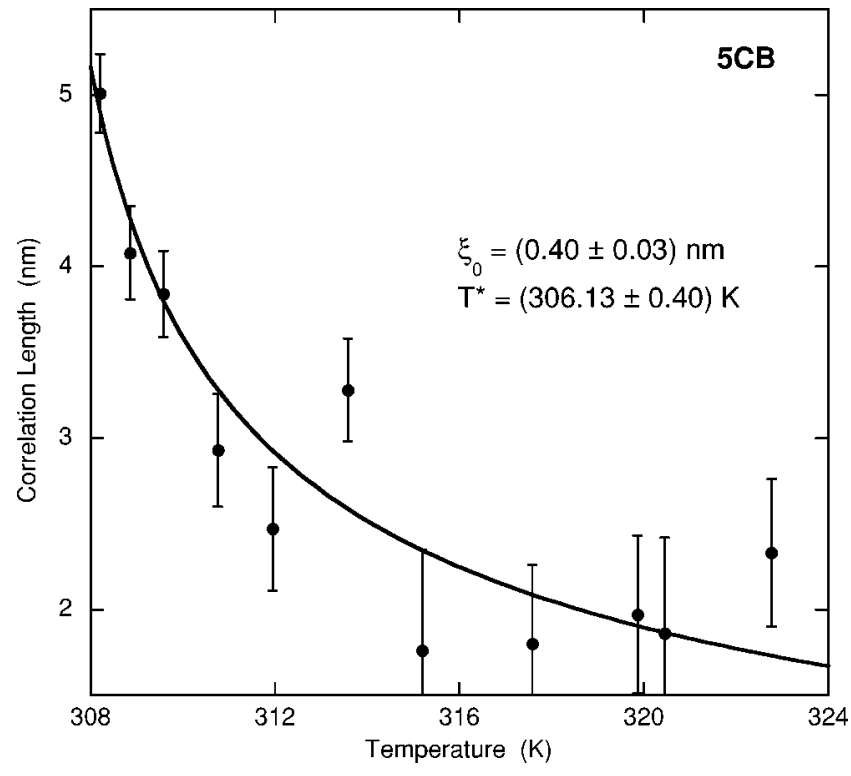

FIG. 3. Correlation length vs. temperature in the isotropic phase of $5 \mathrm{CB}$. The bare correlation length resulting from the fit to Eq. (8) is $0.40 \pm 0.03 \mathrm{~nm}$.

The second compound studied was a chiral-racemic mixture of CE6 with $35 \%$ of the mixture being chiral. This mixture was utilized to allow the chirality to be measured in both the cholesteric and isotropic phases. The CE6 was also obtained from $\mathrm{BDH}$ Limited, and dust was removed with a $0.2 \mu \mathrm{m}$ filter. The cholesteric-isotropic transition temperature was about $318 \mathrm{~K}$, and measurements were made over a range of $5 \mathrm{~K}$ above the transition. The same sample vessel was used and half-wave plates and polarizers were utilized to produce right circular polarized light from the laser and allow only right circular polarized light to enter the detector. There was no evidence that double scattering needed to be taken into account for this more weakly scattering system. From Eq. (7) it is clear that the scattering for CE6 with a right-handed helix comes mainly from the last term for two reasons. First, the denominator of the last term is the least because $q_{0}$ is negative for CE6. Second, for angles greater than $60^{\circ}$ the geometric factors cause the last term to dominate. To a good approximation then, the scattering intensity under these conditions is

$$
I_{\mathrm{RR}}=\frac{D[1+\sin (\theta / 2)]^{4} T}{\left(T-T^{*}\right)\left[1-\xi_{1}^{2} q_{0}^{2}+\xi_{1}^{2}\left(q+q_{0}\right)^{2}\right]} .
$$

This relationship predicts that the scattering intensity at high temperatures where the denominator is very close to $T$ $-T^{*}$ should vary with scattering angle as $[1+\sin (\theta / 2)]^{4}$. This was not quite true, indicating either that the combinations of half-wave plates and polarizers were not acting as perfect circular polarizers or that a very small amount of multiple scattering was present. If either of these causes even a small amount of intensity that varies weakly with scattering angle, it affects the analysis since the theoretical function does not depend strongly on $q$. To overcome this difficulty, the technique of Ref. [4] was utilized, namely, dividing the data at

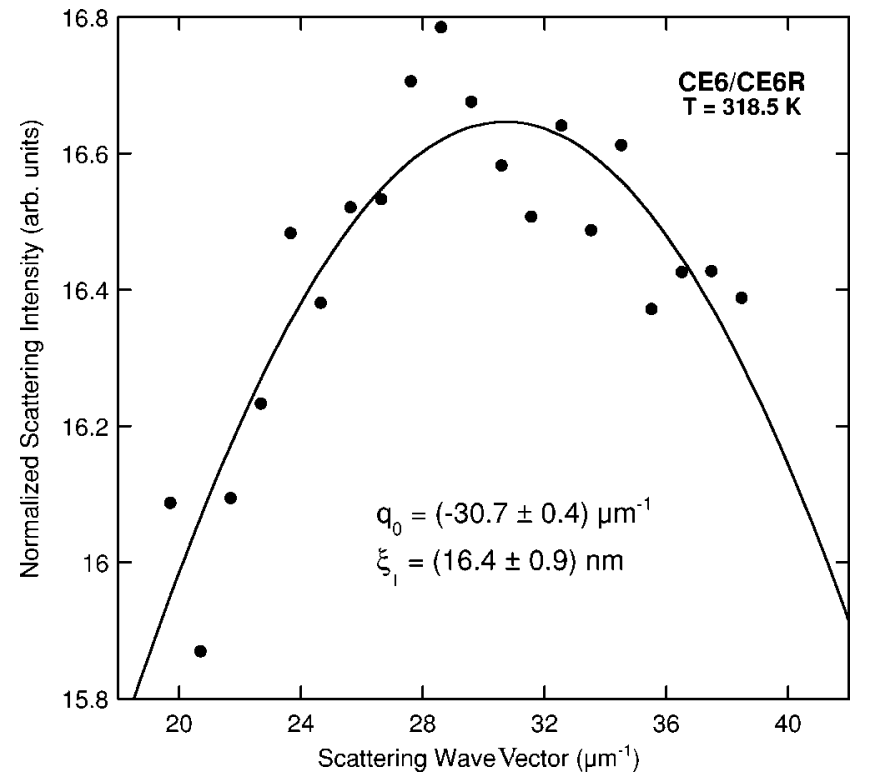

FIG. 4. Scattering intensity normalized to the scattering at high temperature (see text) as a function of scattering wave vector for CE6/CE6R. The line shows the fit to Eq. (13), which results in a determination of the chirality of $q_{0}=-30.7 \pm 0.4 \mu \mathrm{m}^{-1}$.

each temperature by the data taken at $331.5 \mathrm{~K}$, over $13 \mathrm{~K}$ above the transition. Such a technique effectively eliminates all artifacts in the data and should yield data with the angular dependence of Eq. (13) without the $[1+\sin (\theta / 2)]^{4}$ factor. The result of this procedure is shown for one temperature in Fig. 4. Plots of the measured chirality and correlation lengths as a function of temperature are contained in Fig. 5.

In order to compare the chirality of the cholesteric and isotropic phases, measurements were also performed in the cholesteric phase. These were straightforward, involving fabricating a sample (thickness $=10 \mu \mathrm{m}$ ) with the helical axis aligned perpendicular to the glass surfaces (rubbed polyimide surfactant) and measuring the apparent absorption due to selective reflection in a spectrophotometer. The output of the spectrophotometer is shown in Fig. 6 with the sample temperature regulated at $317.3 \mathrm{~K}$. The flat-topped peak is due to selective reflection, and the oscillations are from interference because of the two parallel glass surfaces of the sample cell. The midpoint of the selective reflection peak is equal to the average index of refraction times the pitch. The average index of refraction was measured in an Abbe refractometer in the isotropic phase just above the transition. This measurement was for $589 \mathrm{~nm}$ light, but the refractometer also measured the dispersion so the index at the longer wavelengths of the selective reflection peaks could be determined. The chirality is then $4 \pi$ divided by the pitch. The results of chirality measurements in both the cholesteric and isotropic phases are displayed in Fig. 7. Since the transition temperature as measured in the light-scattering apparatus and spectrophotometer differed slightly, the values of $T_{\mathrm{c}}$ used for the isotropic and cholesteric phase data in Fig. 7 differ by $0.4 \mathrm{~K}$.

\section{DISCUSSION}

The difficulties inherent in measuring the bare correlation length are clear from the few reports that discuss such mea- 


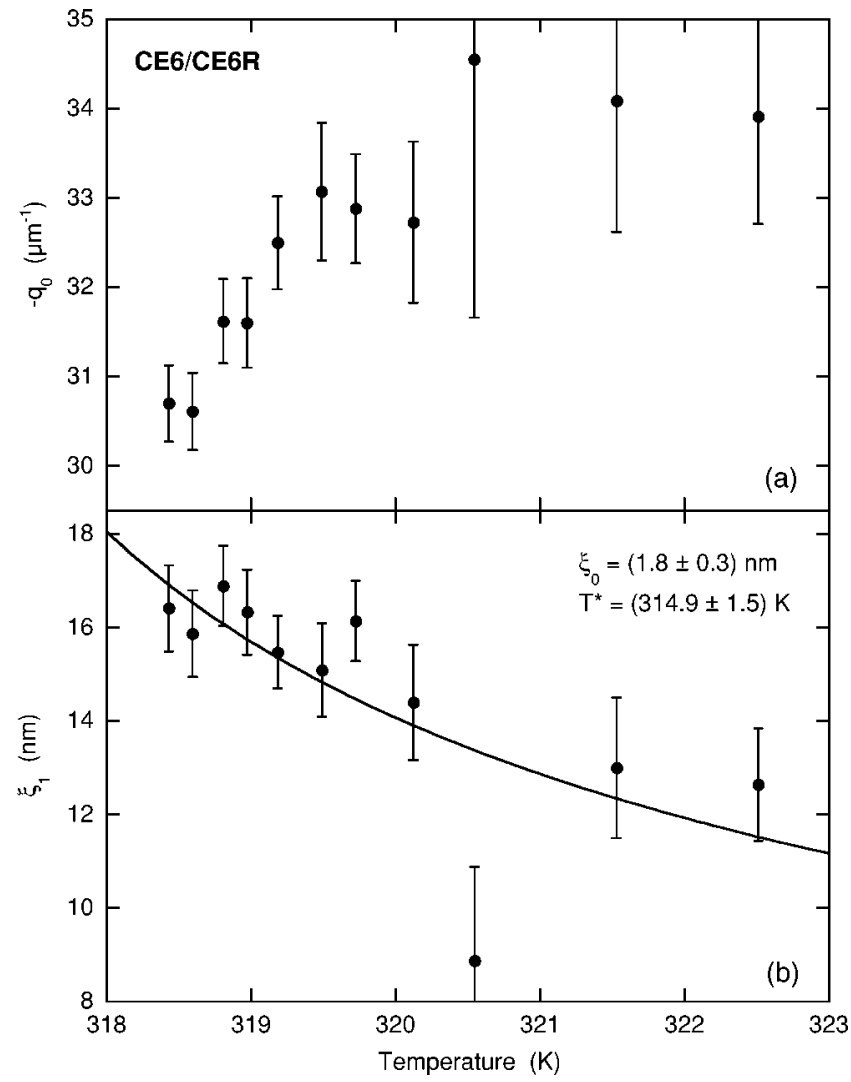

FIG. 5. Chirality (a) and correlation length (b) for the isotropic phase of CE6/CE6R.

surements. The most successful prior investigations used the nonpolar Schiff-based liquid-crystal p-methoxybenzilidinep-butylaniline (MBBA). One report failed to observe the the-

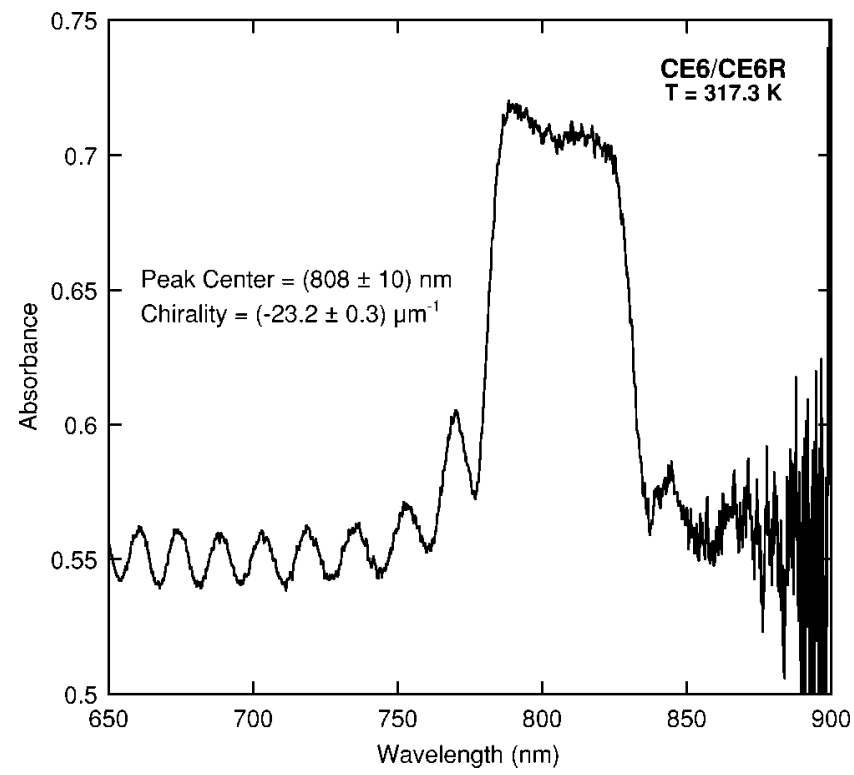

FIG. 6. Wavelength dependence of measured absorption due to selective reflection at one temperature in the cholesteric phase of CE6/CE6R. The small oscillations are due to multiple reflections in the sample chamber, and the noise at the highest wavelengths is due to the decreasing sensitivity of the detector.

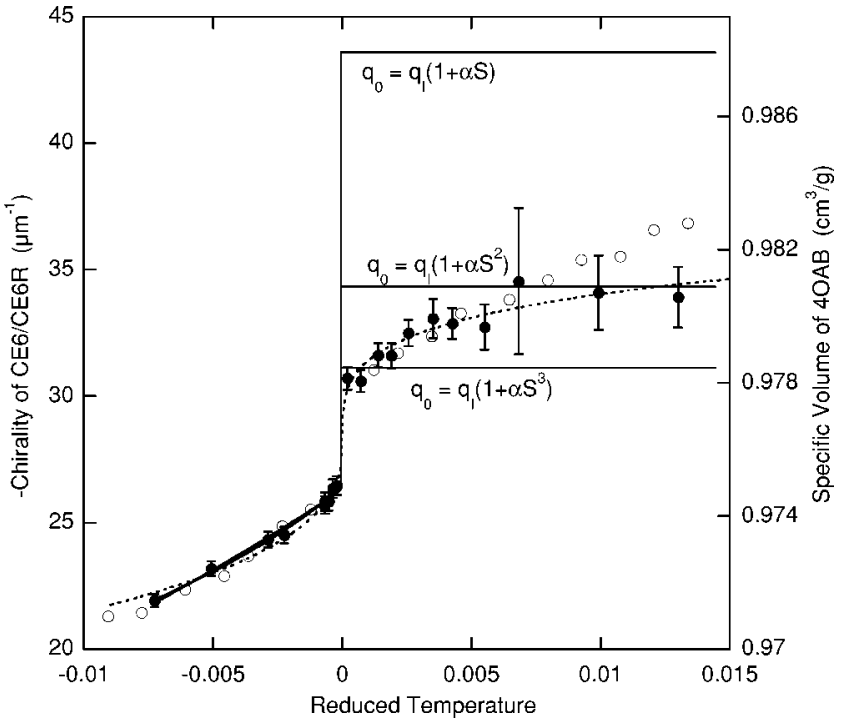

FIG. 7. Chirality as a function of temperature in both the cholesteric and isotropic phases of CE6/CE6R as a function of reduced temperature, $\left(T-T_{\mathrm{c}}\right) / T_{\mathrm{c}}$ (closed circles). Data on the specific volume of $4 \mathrm{OAB}$ from [19] has been graphed for comparison (open circles). The three solid lines are fits of the modified Landau-de Gennes theory (different $q_{0}$ vs $S$ dependences) to the data as described in the text. The dashed curve is a fit of a general critical point theory to the data as also described in the text.

oretical temperature dependence [1], probably due to an experimental artifact. Two successful measurements on MBBA gave conflicting results: $0.68 \pm 0.01 \mathrm{~nm}$ [2] and $0.55 \pm 0.02 \mathrm{~nm}[3]$. The need to remove seemingly insignificant experimental artifacts became very clear in the study of $5 \mathrm{CB}$ reported here. In samples for which the data suggested the presence of an artifact, usually by an extremely small angular dependence of the scattered intensity at high temperatures, analysis of the data was problematic. Whether or not steps were taken to correct for the experimental artifact, the value of the bare correlation length ranged from 0.25 to $0.62 \mathrm{~nm}$. The value reported here, $0.40 \pm 0.03 \mathrm{~nm}$, represents the measurement with the greatest indication of complete artifact elimination and therefore contains no correction step in the analysis.

This result is important for two reasons. First, it is an additional measurement of the bare correlation length in the isotropic phase of a liquid crystal, thus adding precious information to the small number of prior measurements. Second, this is a measurement of the bare correlation length in a polar system that has a strong tendency to form dimers. Therefore, the fact that the value of the bare correlation length is smaller than in MBBA could very well be a reflection of the smaller amount of local orientational order present in systems of molecules with so small a length-tobreadth ratio that they probably would not form a liquidcrystal phase if they did not have a strong tendency to dimerize.

The correlation length measurements that result from the analysis of the CE6 data are roughly three times larger than for $5 \mathrm{CB}$ but decrease with increasing temperature at a rate not unlike what is found for 5CB. This is demonstrated by 
the fit to Eq. (8) shown in Fig. 5, where a significantly larger bare correlation length results and where $T^{*}$ is farther below the first measurement in the isotropic phase. This is consistent with the measurements of the correlation length in a mixture of cholesteryl olyel carbonate and cholesteryl chloride near the Blue Phase III-isotropic phase critical point in which the correlation length is about $18 \mathrm{~nm}, 1.4 \mathrm{~K}$ above the critical point, and increases to $37 \mathrm{~nm}$ very close to the critical point [5]. Since the analysis of the CE6 data requires that the intensity measurements be normalized to data taken at a very high temperature, these results may be slightly less reliable than the 5CB results. But this does not affect the finding that the correlation length in this chiral system is significantly longer than has been measured in nonchiral systems, in agreement with the one prior result.

Figure 7 illustrates several results, all graphed with the independent variable being reduced temperature, $\left(T-T_{\mathrm{c}}\right) / T_{\mathrm{c}}$, where $T_{\mathrm{c}}$ is the transition temperature to the isotropic phase. First, a discontinuity in the chirality at the cholestericisotropic transition of $4.0 \pm 0.6 \mu \mathrm{m}$ or $15 \pm 2 \%$ is present. Second, specific volume (inverse of the density) data from the nematic liquid-crystal dibutyloxyazoxybenzene (4OAB), which has about the same overall molecular shape as CE6, are graphed with the chirality data to show the similarity [19]. Note that the data appear to be quite similar when plotted in this fashion but that the discontinuity in the specific volume is much smaller (about 0.35\%). Third, Fig. 7 also shows that the Landau-de Gennes theory, modified for the cholesteric phase, can be fit to the chirality data in the cholesteric phase. The fitting procedure used is described fully in Ref. [17], but amounts to assuming that the order-parameter behavior of CE6 is typical, leaving only two parameters to be determined, one of which is the chirality in the isotropic phase. Although the theory describes the data well in the cholesteric phase regardless of how the chirality depends on the order parameter, note that different $q_{0}$ versus $S$ dependences predict different discontinuities in chirality at the transition. None of them, however, predicts the observed temperature dependence of the chirality in the isotropic phase.

Finally, as can be seen from Fig. 7, the critical point theory fits the data in both the cholesteric and isotropic phases reasonably well, although rather than a discontinuity of $15 \%$ at the transition, the chirality changes extremely rapidly near the transition with a discontinuity of only $7 \%$. In this fitting procedure, the same dependence of the ordering field on temperature and chiral fraction observed in a very similar cholesteric liquid crystal (CE4) is assumed [14], so only three parameters are left to vary, one of which specifies how far below the critical chiral fraction the system is. The other two parameters describe how much the chirality depends on the ordering and nonordering fields. The result of the fitting procedure is that the chirality depends on the ordering field much more than the nonordering field, but that the system is very close to the critical chiral fraction (the fitting procedure yields $36.4 \% \pm 0.9 \%$ ). Although the data do not rule out a chirality dependence of this kind, the fact that the orientational order parameter changes significantly at the transition and that a coexistence region of about $0.1 \mathrm{~K}$ is present, make it very unlikely that such a strong temperature dependence so close to the transition actually occurs in this system. In addition, since it is clear, experimentally, that $100 \%$ chiral CE6 is still not chiral enough to display a critical point [20], this result points out that even though the theory agrees with the data on either side of the transition, it is not the explanation for the behavior of chirality across the transition. So perhaps the most interesting question raised by this investigation is why the chirality at this very discontinuous transition possesses a relatively small discontinuity and in some ways behaves as if the transition is close to a critical point. The answer may be that chirality, like specific volume but probably less so, is more dependent on short-range rather than long-range interactions, and is therefore affected only weakly by the presence of long-range orientational order. Theoretical work is certainly warranted.

\section{CONCLUSIONS}

Experimental measurements of correlation lengths and chirality in the isotropic phase are exceedingly difficult, but they are important parameters for understanding the physics associated with the transition to the liquid-crystal phase. Correlation lengths in the isotropic phase of nematic liquid crystals have been measured just a few times, always on the same type of compound and with some inconsistency. Even less is known about the chirality of the fluctuations in the isotropic phase above the transition to a cholesteric phase, yet the theories of these fluctuations require knowledge of the chirality. Reported here are correlation lengths for both types of liquid crystals and the chirality of the fluctuations in the isotropic phase for one cholesteric liquid crystal. These results provide useful information: (i) the correlation lengths in a small length-to-breadth ratio polar system are smaller than in a larger length-to-breadth ratio nonpolar system, and (ii) the discontinuity in the chirality across the cholestericisotropic phase transition is relatively small and the chirality in the isotropic phase is temperature dependent. Thus the chirality in some ways resembles the behavior of specific volume at a transition to the isotropic phase or a system just below a critical point more than the prediction of modified Landau-de Gennes theory.

\section{ACKNOWLEDGMENTS}

This work was partially supported by the REU program at the Laboratory for Research in the Structure of Matter of the University of Pennsylvania under NSF Grant No. DMR0079909. J. J. K. acknowledges the support of the Fannie and John Hertz Foundation. Fruitful discussions with P. H. Keyes and T. C. Lubensky are gratefully acknowledged. 
[1] B. Chu, C. S. Bak, and F. L. Lin, Phys. Rev. Lett. 28, 1111 (1972).

[2] T. W. Stinson and J. D. Litster, Phys. Rev. Lett. 30, 688 (1973).

[3] E. Gulari and B. Chu, J. Chem. Phys. 62, 798 (1975).

[4] Y. A. Nastishin, H. Liu, S. V. Shiyanovskii, O. D. Lavrentovich, A. F. Kostko, and M. A. Anisomov, Phys. Rev. E 70, 051706 (2004).

[5] E. P. Koistenen and P. H. Keyes, Phys. Rev. Lett. 74, 4460 (1995).

[6] R. M. Hornreich and S. Shtrikman, Phys. Rev. Lett. 56, 1723 (1986).

[7] D. S. Rokshar and J. P. Sethna, Phys. Rev. Lett. 56, 1727 (1986).

[8] V. M. Filev, Pis'ma Zh. Eksp. Teor. Fiz. 43, 523 (1986).

[9] J. R. L. Rakes and P. H. Keyes, Mol. Cryst. Liq. Cryst. 198, 79 (1991).

[10] L. Longa and H. R. Trebin, Phys. Rev. Lett. 71, 2757 (1993).

[11] L. Longa, W. Fink, and H. R. Trebin, Phys. Rev. E 48, 2296
(1993).

[12] L. Longa, W. Fink, and H. R. Trebin, Phys. Rev. E 50, 3841 (1994).

[13] T. C. Lubensky and H. Stark, Phys. Rev. E 53, 714 (1996).

[14] M. A. Anisimov, V. A. Agayan, and P. J. Collings, Phys. Rev. E 57, 582 (1998).

[15] D. Lacoste, P. J. Collings, and T. C. Lubensky, Phys. Rev. E 65, 031717 (2002).

[16] R. M. Hornreich and S. Shtrikman, Phys. Rev. A 28, 1791 (1983).

[17] B. P. Huff, J. J. Krich, and P. J. Collings, Phys. Rev. E 61, 5372 (2000).

[18] Z. Kutnjak, C. W. Garland, C. G. Schatz, P. J. Collings, C. J. Booth, and J. W. Goodby, Phys. Rev. E 53, 4955 (1996).

[19] R. V. Tranfield and P. J. Collings, Phys. Rev. A 25, 2744 (1982).

[20] G. Voets and W. van Dael, Liq. Cryst. 14, 617 (1993). 